\title{
A importância dos radioprotetores na prática odontológica: uma revisão da
}

\section{literatura}

\section{The importance of radiation protectors in dental practice: a literature review}

Isabella Lopes dos Santos Universidade Brasil, Brasil E-mail: bellalds@hotmail.com

Cristina Toline

Faculdade São Leopoldo Mandic, Brasil E-mail: cristinatoline@gmail.com.br

Beatriz Americano Furuko Universidade Brasil, Brasil

E-mail: bia_furuko@hotmail.com

Beatriz Campolino Schutz Universidade Brasil, Brasil

E-mail: biacampolino@gmail.com

Eduardo de Mattos Fuster Universidade Brasil, Brasil

E-mail: doutoreduardofuster@gmail.com

Irineu Gregnanin Pedron

Universidade Brasil, Brasil

E-mail: igpedron@alumni.usp.br

Michele Cristina Silva do Vale

Universidade Brasil, Brasil

E-mail: neuromvale@gmail.com

Caleb Shitsuka

Universidade Brasil, Brasil

E-mail:cashitsuka@gmail.com

\begin{abstract}
Resumo
Os exames de imagem fazem parte do cotidiano do Cirurgião-Dentista em sua prática clínica, por isso é importante o conhecimento dos cuidados relacionados a radioproteção, desta forma evitamos exposições desnecessárias aos Raios$\mathrm{X}$. Este trabalho tem por objetivo apresentar através de uma revisão de literatura, a respeito dos os principais radioprotetores utilizados em odontologia. Os artigos foram pesquisados nas bases de dados PubMed, Bireme, Scielo e Lilacs. Os critérios de exclusão foram a data de publicação, e relação com o tema proposto pelos descritores. Após avaliação, foram selecionados 27 artigos os quais constituem o corpo bibliográfico desta revisão. Conclui-se que é necessário maior exploração do tema diante da complexidade e importância acerca do uso dos radioprotetores, sugerindo uma abordagem mais aprofundada ainda nos cursos de graduação.
\end{abstract}

Palavras-chave: Radiologia; Radiologia odontológica; Odontologia; Radioproteção; Raios X.

\begin{abstract}
Imaging exams are part of the dentist's daily life in their clinical practice, so it is important to know the care related to radioprotection, thus unnecessary exposure to X-rays. The scope of this research aims to present, through a literature review, the main radiation protectors used in dentistry. Articles were searched in PubMed, Bireme, Scielo and Lilacs databases. Exclusion criteria were the date of publication and relationship with the topic proposed by the descriptors. After evaluation, 27 articles were selected, which constitute the bibliographic body of this review. Conclusion: It is concluded that further exploration of the topic is necessary in view of the complexity and importance of the use of radiation protectors, suggesting an even deeper approach in undergraduate courses.
\end{abstract}

Keywords: Radiology; Dental radiology; Dentistry; Radiation protection; X Ray.

\section{Introdução}

A solicitação e aplicação de exames radiográficos complementares objetivando o diagnóstico do paciente, é uma prática rotineira para o cirurgião-dentista. (Villalobos et al., 2021). Contudo, para Alves, et al, 2016; Cirilo, 2021, Lima et al, 
2021, a radiação ionizante pode ser extremamente nociva para pacientes, e para profissional, tornando imprescindível o dominío a respeito das formas de radioproteção existentes.

Os raios-X foram descobertos em 1895, pelo físico alemão, Wilhelm Conrad Röntgen, após constatar que durante o tempo de continuidade de descarga elétrica incidida sobre a ampola, uma luz incadescente era reproduzida sobre uma tela fosforescente que estava presente no laboratório. Percebeu também, que, mesmo que existissem obstáculos entre a ampola, e a tela, a claridade se matinha, concluindo então que essa energia era capaz de penetrar por diferentes espessuras, revelando imagens. (Costa, 2013, Santos, 2021).

Os exames de imagem contribuem significativamente na elaboração e confirmação de diagnósticos, todavia, é necessário o estudo e conhecimento a cerca dos riscos advindos da exposição acidental, ou repetida a radiação ionizante. A literatura descreve que o uso inadivertido, ou excessivo das radiações ionizantes podem trazer danos aos tecidos biológicos e celular, levando a um prejuízo permanente, promovendo alterações moleculares no DNA, possíveis mutações, e morte das células. (Costa, 2013; Costa et al., 2021; Okuno, 2013; Santana, 2017; Santos, 2021).

No ambiente do consultório odontológico, o cirurgião-dentista é o responsável pela radioproteção dos pacientes e funcionários de acordo com as diretrizes da Portaria $n^{\circ}$ 453/98. Desta forma, se faz necessária a discussão a respeito da importância da radioproteção na prática odontológica-, visto que a exposição acidental a essa onda eletromagnética, é capaz de gerar efeitos colaterais permanentes bastante expressivos. (Cirilo, 2021; Costa, 2013, Lima et al, 2021).

\section{Metodologia}

Esta revisão bibliográfica da literatura avaliou artigos publicados de 2002 a 2021, relacionados a importância da radioproteção durante os exames de Raio-X em odontologia. Inicialmente foram selecionados 31 artigos nas bases de dados como a PubMed, Bireme, Scielo e Lilacs. Os critérios de exclusão foram baseados na data de publicação, e relação com o tema proposto pelos descritores. Após análise, foram selecionados 27 artigos que foram os quais constituem o corpo bibliográfico desta revisão (Estrela, 2018).

\section{Resultados e Discussão}

Pensando em radioproteção, é importante obedecer ao princípio da justificação, que se baseia em evitar o uso desnecessário de radiação ionizante, por meio do esclarecimento do motivo pelo qual cada tomada que será realizada. Esse princípio tem como prerrogativa, que o benefício da exposição a radiação ionizante, deve ser maior que o possível dano que a radiação possa causar, sempre pautados na dose mínima. (Leyton et al, 2014).

Todos os seres humanos são expostos diariamente a presença de radiação natural, e artificial; A respeito das radiações ionizantes artificiais para exames diagnóticos, as doses utilizadas para obtenção de radiografias pelos cirurgiões-dentistas são relativamente baixas quando comparadas com as doses de radiação provindas de fontes naturais que um ser humano pode receber em média no período de um ano. A literatura sugere que a exposição por radiação de fontes naturais tem valores de aproximadamente 2,4mSv/ano, em contrapartida, a dose de radiação de uma radiografia periapical é de aproximadamente 0,001 a 0,008mSv. Os efeitos deletérios da exposição repetida a radiação ionizante, são variáveis, e dose- dependentes, ou seja, estão relacionados com o indivíduo, e a dose por ele recebida. Os efeitos colaterais tardios da exposição aos raios-X são classificados em dois conceitos: Efeitos determinísticos, e efeitos estocásticos, conforme descrito por (Alves et al, 2016; Costa, 2017; CRO-SP, 2014, Santos, 2021).

Os efeitos estocásticos, estão relacionados proporcionalmente à dose de exposição, podendo levar ao surgimento de catarata radiogênica, radiodermite e esterilidade. Contudo, a gravidade dos efeitos, é independente da dose recebida, criando 
um padrão inversamente proporcional. Já os efeitos determinísticos são dependentes de uma alta radiação ionizante total, ou localizada de um tecido, e se dividem em dois subgrupos: os efeitos hereditários, e os efeitos somáticos. (Leyton et al, 2014; Okuno, 2013; Oliveira et al, 2016; Xavier et al, 2014).

Okuno, (2013), cita que os efeitos somáticos são aqueles que acometem o próprio indivíduo que recebeu a radiação, enquanto os efeitos hereditários, irão se manifestam nos descendentes do indivíduo irradiado, devido a danos causados nas células de ordem reprodutora deste.

Após a interação célula com a radiação ionizante, a energia residual ocorre por meio da irradiação direta, e indireta. A irradiação indireta é conceituada como o momento em que a radiação entra em contato com as moléculas, ocasionando mutações de ordem genética no DNA, capaz de levar a morte celular. A irradiação indireta tem início quando acontece o processo de radiólise da água, levando a produção de radicais livres que podem alcançar outras importantes moléculas, tornando-as ionizadas. (Santos, 2021; Okuno, 2013).

Após serem percebidos os possíveis efeitos deletérios inerentes ao uso de radiação para fins de diagnósticos médicos ou odontológicos, foi criada em 1 de junho de 1998, a legislação da Portaria SVS/MS n 453, que discorre a cerca dos riscos, da exposição à radiação ionizante. Contudo, em 20 de dezembro de 2019, a ANVISA, revogou este documento mediante a apresentação da Resolução- RDC N³30, que veio a se tornar um dos documentos referenciais para atendimentos em práticas radiológicas. Este documento trata de modificações importantes a respeito de pontos como: o modo, e padronização do uso do dosímetro, definições, e adoção de níveis de dose referidas na CNEN (Comissão Nacional de Energia Nuclear). (Gomes, 2012; Costa, 2013; Brasil, 2019).

Os riscos relacionados a exposição aos exames de imagem radiológica, são considerados mínimos, quando seguidos todos os protocolos de radioproteção. De acordo Alves et al. (2016) e Costa (2017), com relação aos exames realizados em odontologia, quando nos referimos aos níveis de radiação emitidos, o risco se torna ínfimo, quando observados todos os protocolos de segurança.

Conforme Lima et al., (2021), o local do consultório odontológico onde ocorre a emissão de radiação ionizante, deve estar de acordo com a Portaria $n^{\circ}$. 453/98, que orienta sobre a obrigatoriedade de sinalização dos ambientes por meio de quadros de orientação sobre radioproteçãe correto local para armazenamento dos protetores plumbíferos.

Segundo os parâmetros sugeridos pela RCD 330, os protetores plumbíferos individuais devem apresentar uma espessura mínima de $0,25 \mathrm{~mm}$ de chumbo, e proteger a extensão de tronco por completo, assim como a área de tireoide, além das gônadas durante a exposição à radiação ionizante. Soares, (2014), e Gomes, (2012), sugerem que a ideia sobre o correto do uso de aventais plumbíferos para atenuação da radiação ionizante, é um assunto atual, e necessário, visto que o seu desconhecimento, ou prática inadequada pode acarretar grandes prejuizos a curto, e longo prazo.

O protetor plumbífero individual com espessura $(0,25 \mathrm{mmPb})$, na tensão de $75 \mathrm{kV}$ foi decisivo na atenuação das doses de radiação ionizante no paciente durante a exposição em até 95\%. Apesar disso, a comunidade ciêntifica aponta falhas sobre o modelo correto de armazenamento do protetor individual de chumbo. Os achados inferem que eles devem ser acondicionados de forma que fiquem estendidos, pois, caso sejam dobrados, ou amassados, o fator de proteção será prejudicado, devido a formação de finas fendas na camada de chumbo, comprometendo diretamente a atenuação dos feixes do raio-X. (Gomes, 2012; Oliveira, 2016; Soares, 2014).

Para a proteção no ambiente físico, é essencial que haja uma blindagem protetora na sala de raios-X, proveniente do revestimento das paredes com argamassa misturada a barita, que opera como obstáculo dos feixes da radiação ionizante, sendo indispensável para a proteção do profissional, e técnicos em radiologia durante a exposição. A barreira de proteção física não tem a habilidade de embargar a transmissão, da radiação, e sim de atenuá-la, quando devidamente realizados os cálculos 
dosimétricos importantes para a correta blindagem da sala. Essa eficiência de blindagem é obtida por meio do levantamento radiográfico da sala, e a radiometria. Os níveis devem obedecer aos padrões exigidos pela Agência Nacional de Vigilância Sanitária (ANVISA), de acordo com a portaria federal $n^{\circ} 453 / 98$, sendo realizada por profissionais capacitados. A RDC 330/2019 preconiza que tratando-se de proteçao radiológica para exposições ocupacionais, que a dose máxima mensal não ultrapasse, $20 \mathrm{mSv}$, durante um período de até 5 anos. Desta foma caso a carga trabalhista seja excedente a $30 \mathrm{~mA} / \mathrm{min}$, é obrigatório que o profissional se mantenha protegido por barreira física com ao menos, 0,5 mm, o que o torna equivalente ao chumbo. Sobre os exames odontológicos intraorais, é aconselhałevel que, o operador se posicione a uma distância de 2 metros do tubo do aparelho. O dosímetro, é obrigatorio para consultórios odontológicos que possuam mais de um aparelho de raio-X. (Costa, 2013; Muniz et al, 2021).

Ferreira et al., (2021), avaliaram por meio de foi um questionário autoaplicável, físico e digital, 85 alunos distribuídos no $1^{\circ}$, e $4^{\circ}$ período do curso de odontologia do Centro Universitário UNIFAMETRO, (Fortaleza - CE). Os resultados sugerem que, $91 \%$ dos estudantes compreendem o conceito de biossegurança, entretanto, é em relação a como proceder em caso de acidentes nos laboratórios/clínicas, que os achados se mostram mais significantes, visto que $88 \%$ dos alunos do $1^{\circ}$ período não saberiam com agir nessa situação., Já na turma do $4^{\circ}$ período os dados se mostram alarmantes pois, $74 \%$ dos alunos possui dúvidas, e questionamentos a respeito desse tema. Esse resultado demonstra a necessidade de um aprofundamento no ensino e prática das questões a respeito da radioproteção nos cursos de graduação. (Ferreira et al, 2021).

Souza e colaboradores, (2020), apresentam o uso de uma ferramenta digital (RADIOAPP), com questões e textos sobre radiologia, e radioproteção para os alunos do curso de Odontologia da Universidade Luterana do Brasil (ULBRA), no período de 2017 a 2018. Esse aplicativo foi desenvolvido com o intuíto de agregar conhecimento aos graduandos, ao mesmo tempo que fornece um material didático, com acesso em tempo integral. Concluem ainda que o uso de ferramentas digitais, podem aprimorar o conhecimento dos acadêmicos em relação a radiologia, e radioproteção em odontologia. (Souza et al, 2020).

Sousa et al., (2020), verificaram a aderência de 52 alunos do curso de odontologia da UEPB aos protocolos de biossegurança durante a execução de técnicas radiográficas. Verificou-se por meio da observação que 40,38\% dos alunos conseguiram realizar os procedimentos de forma segura sem o auxílio dos professores, entretanto, 63,46\% dos alunos não orientaram seus pacientes no momento que antecedeu o exame, 98,07\% dos pacientes usaram avental plumbífero durante a tomada radiográfica, o que aconteceu em apenas 90,38\% dos pacientes sobre o uso do protetor de tireóide. Tais resultados inferem que apesar de os alunos terem conhecimento, e fazerem uso das técnicas de radioproteção durante o uso de Raios-X, é necessário que esse tema seja abordado de forma incisiva nos cursos de graduação em odontologia. (Sousa et al, 2020).

Santana, e colaboradores, (2017), corroboram com a necessidade de utilização do protetor de tireoide, após constatarm que uma grande parcela dos cirurgiões-dentistas faz uso dos aventais de chumbo, entretanto, apenas uma pequena parcela utiliza somente do protetor de tireoide, todavia a literatura sugere o uso dos aventais e protetores de tireóide para que se obtenha uma proteção adequada. (Santana et al, 2017).

Alves et al., (2016), apresentam em seu estudo a relação entre os conhecimentos e métodos sobre radioproteção aplicados por cirurgiões-dentistas em sua prática clínica e verificou-se que $100 \%$ dos profissionais tem preocupação aparente com a técnicas e métodos de proteção, todavia, apenas $92 \%$ destes fazerem uso de posicionadores radiográficos, enquanto $96 \%$ relatam fazer uso de aventais plumbíferos, somente $92 \%$ fazem uso do protetor de tireóide. A respeito das proteções físicas do ambiente, Alves et al., (2016) constatou que, apenas 38\% possuem proteção de chumbo nas paredes de seus consultórios, e aliado a isso somente $20 \%$ dos profissionais fazem uso de aventais de proteção em si mesmos. Esses dados apontam que mesmo que o cirurgião-dentista conheça os riscos e fatores de radioproteção, uma grande parcela não faz uso de todas 
possibilidades de forma adequada, o que reflete na necessidade e importância de um aprofundamento desta disciplina nos cursos de graduação, e especialização. (Alves et al, 2016).

O ensino de Odontologia, presencial ou remotamente, deve ter maior atenção ao que se refere as normas vigentes, e diretrizes de radioproteção, para que sejam aplicadas na prática profissional durante o atendimento odontológico. (Lima et al., 2021; Maltarollo et al., 2021; Valente et al., 2021; Rocha et al., 2021)

\section{Considerações Finais}

Conclui-se que o cirurgião-dentista, é o principal responsável por difundir o conhecimento em relação as normas de proteção à sua equipe e também orientar seus pacientes quanto a importância do uso. Desta forma, se torna indiscutível a necessidade de constante atualização ao que se refere a legislação. Sugere ainda um maior aprofundamento no ensino nos cursos de graduação ao que se refere as normas vigentes, e diretrizes de radioproteção.

\section{Referências}

Alves, W. A, Camelo, C. A. C, Guaré, R. O, Helena, C, Costa, M, \& Almeida, M. S. C. (2016). Radiological protection: knowledge and methods of dentist. Arq. Odontol. 52(3), 130-135.

Brasil. (2019). RCD-330. Ministério da Saúde.

Brasil. Congresso. Senado. Projeto de lei 3661, de 10 de julho de 2002. Exercício das profissões de técnico e tecnólogo em radiologia e de bacharel em ciências radiológicas. https://www.camara.leg.br/propostas-legislativas/540959.

Cirilo, A. N, Neto, D. P, Tessaro, G. C, Vilarim, L. T, \& Filgueiras, P. S. (2021). Radiação ionizante: Uma revisão de literatura. Revista acadêmica Novo Milênio. 3(4).

Costa, M. M. de O, Santos, K. R. do N, Oliveira, F. M. de, \& Costa, D. H. (2021). Alerta sobre a importância do conhecimento das radiações ionizantes e uso de protetores plumbíferos na radiologia odontológica. E-Acadêmica, 2(3), e092348. https://doi.org/10.52076/eacad-v2i3.48

Costa, T. (2013). Os riscos provenientes da radiologia odontológica. Sociedade brasileira de proteção radiológica - sbpr. 15(19), 01-05.

CRO/SP. (2014). Câmara Técnica Radiologia Odontológica. Radiologia ou Imaginologia Odontológica.

Estrela, C. (2018). Metodologia Cientifica, Ensino e Pesquisa. Editora Artes Médicas.

Ferreira, I. C., da Silva, I. A. G., Andrade, L. G. N., Leite, J. J. G., \& da Silveira, P. V. (2021). Avaliação do conhecimento acerca da biossegurança em estudantes de odontologia. Revista Expressão Católica Saúde, 6(1), 20-25.

Gomes, C. K, (2012). Avaliação do conhecimento dos usuários da faculdade de odontologia da UFJF quanto às medidas de radioproteção. Odontol. Clín.Cient. 11(1), 25-29.

Leyton, F, Canevaro, L, Dourado A, Castello, H, Bacelar, A, Navarro, M. T, Vãno, E, Nogueira, M. S, Batista, W. O, Furquim, T. A. C, Lykawka, R, Melo, C. S, Borges, F, \& Rodrigues, B. (2014). Riscos da Radiação X e a Importância da Proteção Radiológica na Cardiologia Intervencionista: Uma Revisão Sistemática. Rev. Bras. Cardiol. Invasiva. 22(1), 87-98.

Lima, B. N. S, Santos, J. V. S. dos, Morais, B. D, Meireles, A. C. N, Silva Filho, W. J., Santos, M. A. L. dos, Couto, G. R, Menezes, L. dos S, \& Takeshita, W. M. (2021). O PBL (Problem Based Learning) para a disciplina de Radiologia Odontológica é aplicável? Uma revisão sistemática. Research, Society and Development, 10(9), e1410917140. https://doi.org/10.33448/rsd-v10i9.17140

Lima, R., Santos, P., Hora, I., \& Gutierrez, G. (2021). Radiação ionizante na odontologia, quais os cuidados devemos ter? Jornada odontológica dos acadêmicos da católica, 6. http://reservas.fcrs.edu.br/index.php/joac/article/view/4520

Lima, R, Santana, V, Hora, I, \& Gutierrez, G. (2021). Minimizando os riscos físicos na odontologia: o que devo fazer? Jornada odontológica dos acadêmicos da católica, 6. http://publicacoesacademicas.unicatolicaquixada.edu.br/index.php/joac/article/view/4509/4006.

Maltarollo, T. F. H, Domingues, L. de O, Pedron, I. G, Hughes Júnior, G. B, Pais, K. dos S, Araújo, G. P. M. C. de, \& Shitsuka, C. (2021). Manejo do desgaste dentário erosivo. E-Acadêmica, 2(3), e112346. https://doi.org/10.52076/eacad-v2i3.46

Muniz, F, da Silva Barbosa, J, da Silva, H. F. V, da Silva, T. V. S, de Lima, M. P, de Assunção, M. G, \& Suassuna, F. C. M. (2021). Dose de radiação absorvida pelo operador utilizando aparelhos de raios $\mathrm{x}$ odontológicos portáteis: Uma revisão integrativa da literatura. Research, Society and Development, 10(9), e48310918082-e48310918082.

Okuno, E. (2013). Efeitos biológicos das radiações ionizantes. Acidente radiológico de Goiânia. Estudos avançados, 27(77), 185-199. 
Oliveira, D. H. M, Almeida, M. S. C, Costa, C. H. M, \& Sousa filho, L. F. (2016). Meios de proteção contra radiação utilizados em estabelecimentos de assistência à saúde odontológica. RFO UPF. 21(2), 167-171.

Rocha S. S. D, Joye C. R, Moreira MM. (2020) A Educação a Distância na era digital: tipologia, variações, uso e possibilidades da educação online. RSD. 9(6):e10963390. https://rsdjournal.org/index.php/rsd/article/view/3390

Santana A. S., Moura, J. R, \& Silva, N, M. (2017). Os serviços odontológicos e as medidas de radioproteção Dental services and radioprotection measures. RFO. 22(2).236-242.

Santos, P. S. (2021). O mau uso dos epi's em radiologia por profissionais de saúde: Uma revisão de literatura. Repositório institucional Faculdade Maria Milza. http://131.0.244.66:8082/jspui/handle/123456789/2270.

Soares, F. A. P. (2014). Utilização de vestimentas de proteção radiológica para redução de dose absorvida: uma revisão integrativa da literatura. Radiol bras. 44(2), 97-103.

Souza, J. F, Machado, G. M, Oliveira, D, Wiltgen, A, Mahl, C. R. W, Brew, M. C, \& Bavaresco, C. S. (2020). Criação de um aplicativo para área de radiologia odontológica: qualificando o ensino em nível de graduação. Brazilian Journal of Development, 6(10), 80250-80260.

Sousa, R. P. R., Andrade, F. F, dos Santos, K. R. G, da Silva Fonseca, F. M., de Farias Freitas, A. P. L., Dantas, D. C. R. E., \& Diniz, D. N. (2020). Avaliação das medidas de biosegurança na execução da técnica radiografica intrabucal durante procedimentos clínicos e endodônticos. Brazilian Journal of Health Review, 3(5), 13717-13727.

Valente G. S. C, Moraes E. B de, Sanchez M. C. O, Souza D. F, \& Pacheco M. C. M. D. (2020) O ensino remoto frente às exigências do contexto de pandemia: Reflexões sobre a prática docente. $R S D$. 9(9):e843998153. https://rsdjournal.org/index.php/rsd/article/view/8153

Villalobos, M. I. O. \& B, Martorano, J. F, Juhás, R, \& Nakao, E. (2021). Proposta de protocolo para solicitação de exames de imagens odontológicas. Research, Society and Development, 10(7), e33210716573. https://doi.org/10.33448/rsd-v10i7.16573

Xavier, A. M, Gaidano, E, Moro, J. T, \& Heilbon, P. F. (2014). Princípios básicos de segurança e proteção radiológica. e-radiologia. 4 , $27-28$. 